\title{
Editorial
}

\section{High-Performance Computing Strategies for Complex Engineering Optimization Problems}

\author{
Gongnan Xie, ${ }^{1}$ Massimo Scalia, ${ }^{2}$ Masoud Rokni, ${ }^{3}$ Balaji Raghavan, ${ }^{4}$ and Manyu Xiao ${ }^{5}$ \\ ${ }^{1}$ School of Mechanical Engineering, Northwestern Polytechnical University, Shaanxi, Xian, China \\ ${ }^{2}$ Department of Mathematics, University of Rome "La Sapienza", Piazzale Aldo Moro 5, 00185 Rome, Italy \\ ${ }^{3}$ Department of Mechanical Engineering, Technical University of Denmark, Building 403, Room 115, 2800 Kongens Lyngby, Denmark \\ ${ }^{4}$ Civil Engineering Laboratoire de Génie Civil et Génie Mécanique INSA Rennes, France \\ ${ }^{5}$ Department of Applied Mathematics, Northwestern Polytechnical University, Shaanxi, Xian 710072, China
}

Correspondence should be addressed to Gongnan Xie; gongnan.xie@gmail.com

Received 10 September 2014; Accepted 10 September 2014; Published 29 December 2014

Copyright (C) 2014 Gongnan Xie et al. This is an open access article distributed under the Creative Commons Attribution License, which permits unrestricted use, distribution, and reproduction in any medium, provided the original work is properly cited.

Managing computational effort (CPU time, memory, and interfacing) is a major issue in engineering design optimization problems due to the cost of the high-fidelity numerical simulations (finite elements, finite volumes, etc.) involved. In order to decrease the overall cost of the optimization process, parallel algorithm, reduced-order models, or their combinations are an economical and efficient option.

The main goal of this special issue is to bring together theoretical, numerical, and experimental contributions that describe original research results and/or innovative concepts that address all aspects of complex engineering optimization problems. In this issue we received 62 paper submissions and finally 30 original research papers on the frontier of high-performance computing strategies were accepted for publication based on critical peer-review process. This issue covers a wide range of topics in solid mechanics, thermodynamics, fluid mechanics, and advanced computing or optimization methods. We hope that these topics could be continued to track the updated trends year by year.

\section{Acknowledgments}

We would like to express our thanks to all the contributors of this special issue for their support and cooperation and to qualified reviewers for evaluating paper quality of this special issue.

Gongnan Xie Massimo Scalia Masoud Rokni Balaji Raghavan Manyu Xiao 


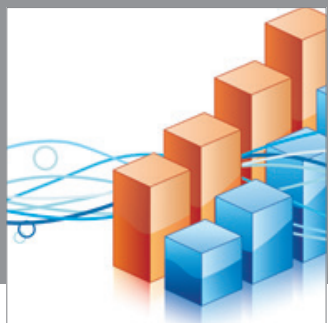

Advances in

Operations Research

mansans

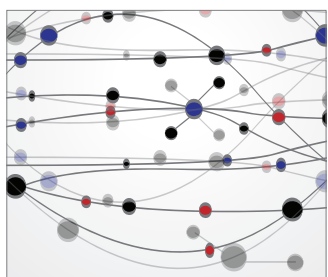

The Scientific World Journal
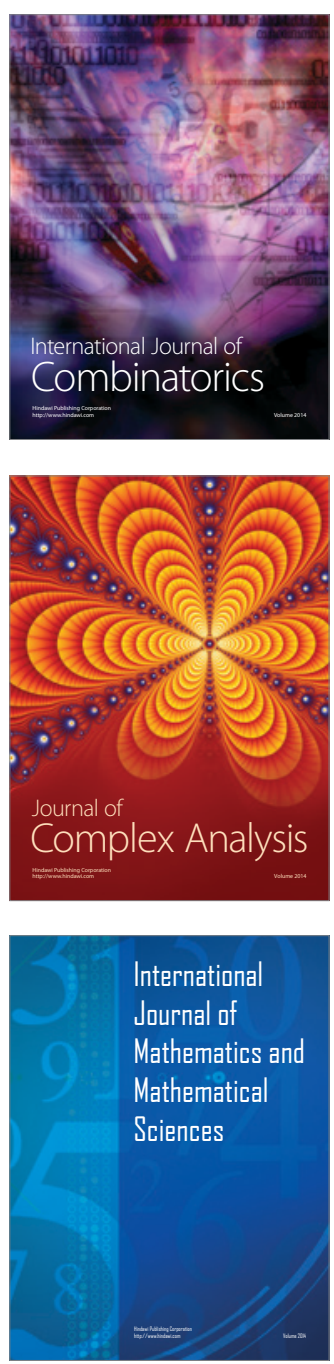
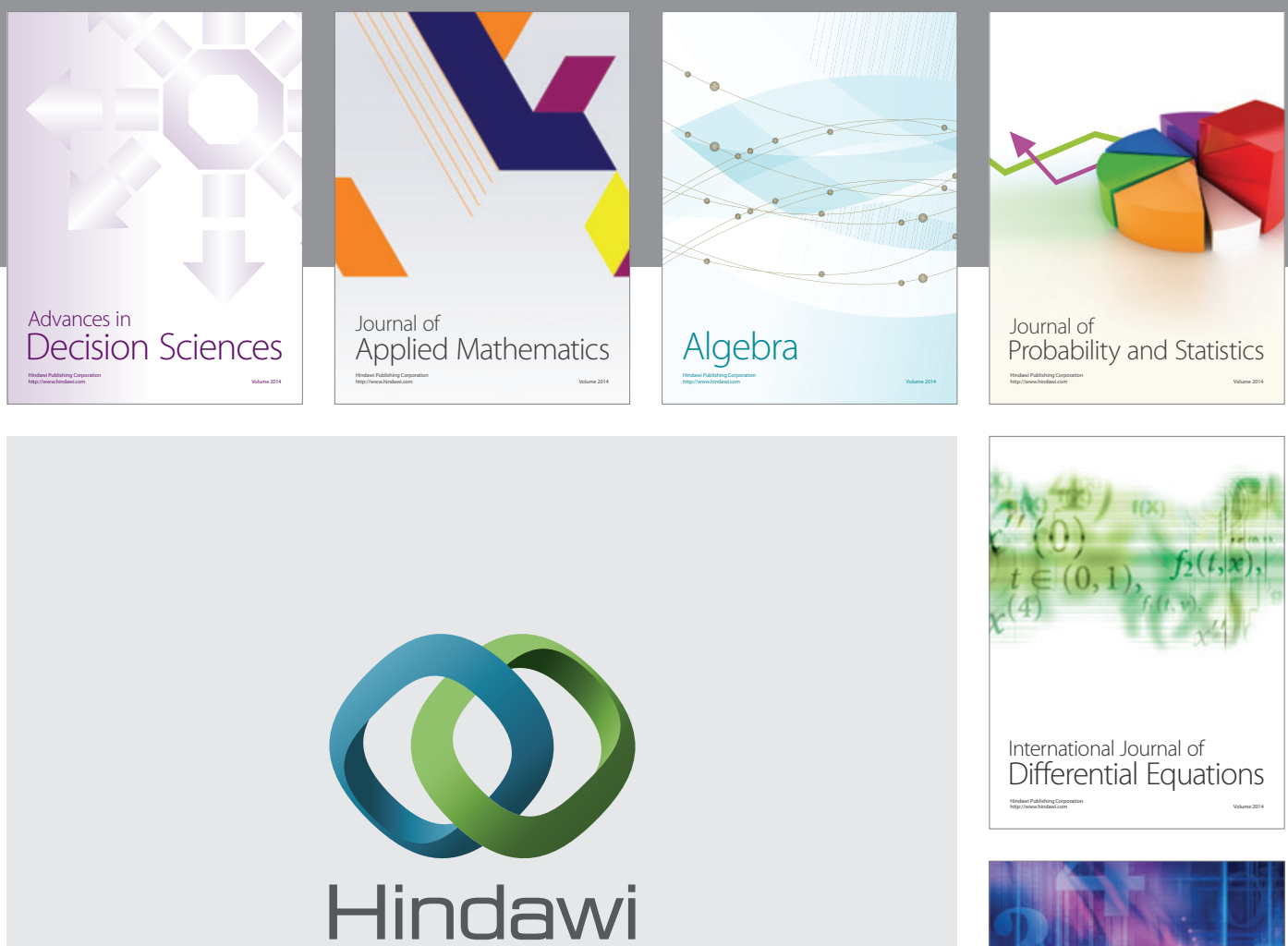

Submit your manuscripts at http://www.hindawi.com
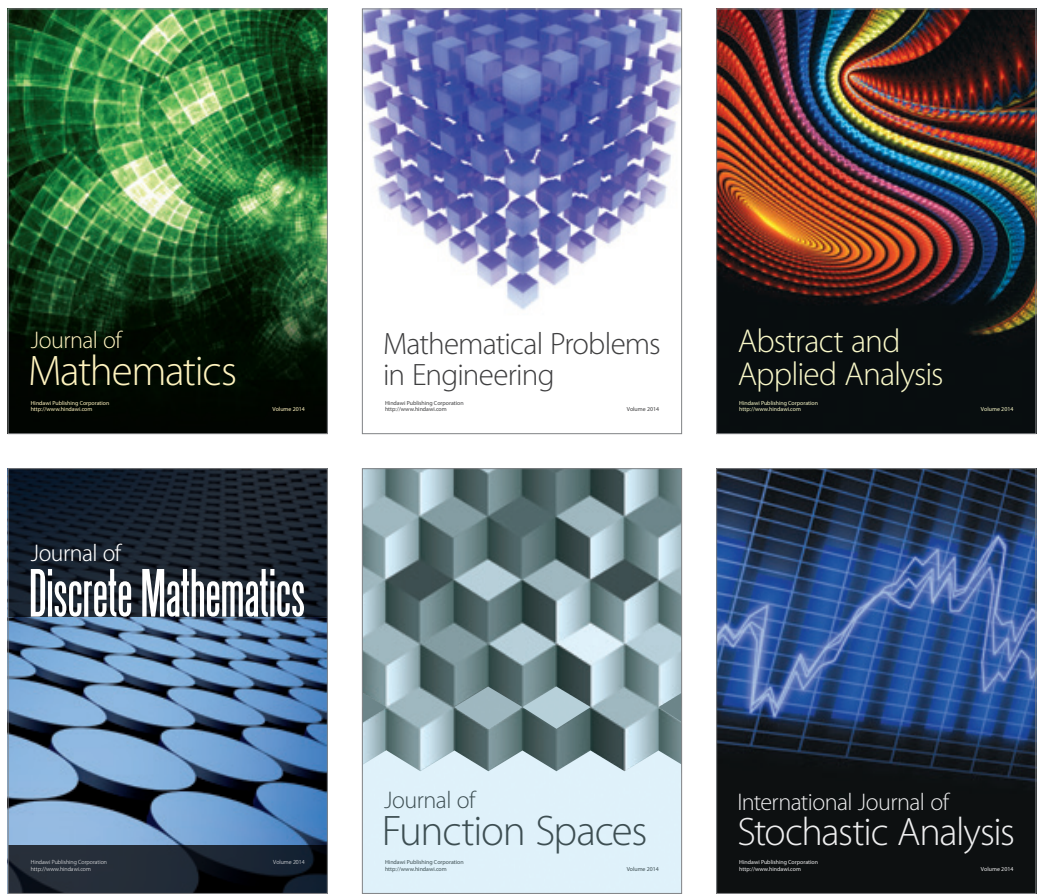

Journal of

Function Spaces

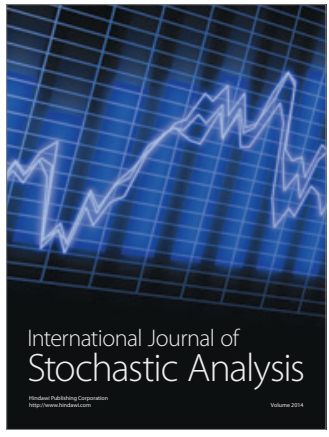

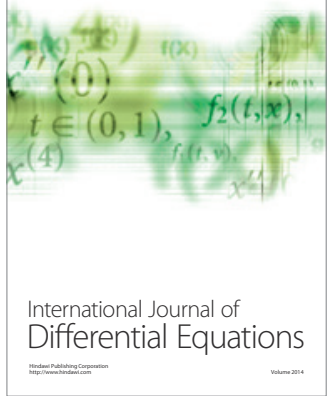
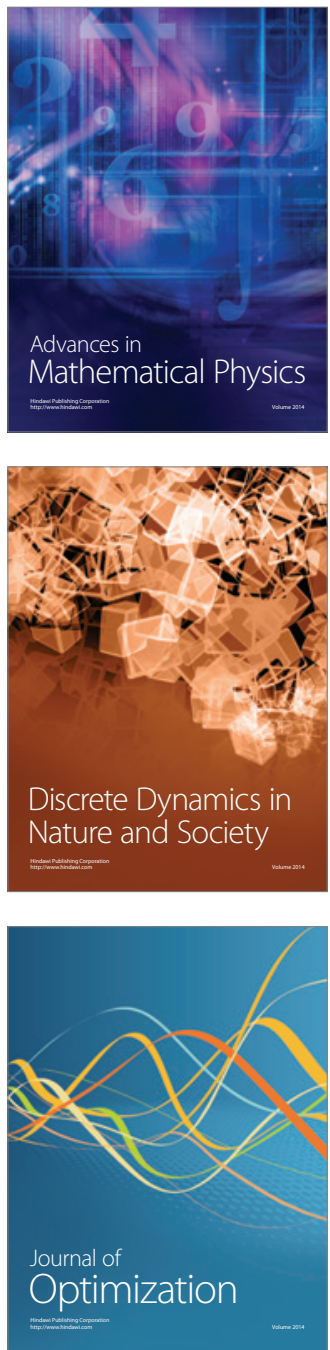